\title{
EXPANDING AIRSTAR CAPABILITY FOR FLIGHT RESEARCH IN AN EXISTING AVIONICS DESIGN
}

\author{
Sean A. Laughter, NASA Langley Research Center, Hampton, VA
}

\begin{abstract}
The NASA Airborne Subscale Transport Aircraft Research (AirSTAR) project is an Unmanned Aerial Systems (UAS) test bed for experimental flight control laws and vehicle dynamics research. During its development, the test bed has gone through a number of system permutations, each meant to add functionality to the concept of operations of the system. This enabled the build-up of not only the system itself, but also the support infrastructure and processes necessary to support flight operations. These permutations were grouped into project phases and the move from Phase-III to Phase-IV was marked by a significant increase in research capability and necessary safety systems due to the integration of an Internal Pilot into the control system chain already established for the External Pilot.
\end{abstract}

The major system changes in Phase-IV operations necessitated a new safety and failsafe system to properly integrate both the Internal and External Pilots and to meet acceptable project safety margins. This work involved retrofitting an existing data system into the evolved concept of operations. Moving from the first Phase-IV aircraft to the dynamically scaled aircraft further involved restructuring the system to better guard against electromagnetic interference (EMI), and the entire avionics wiring harness was redesigned in order to facilitate better maintenance and access to onboard electronics. This retrofit and harness re-design will be explored and how it integrates with the evolved Phase-IV operations.

\section{AirSTAR Overview}

The Airborne Subscale Transport Aircraft Research (AirSTAR) project is part of NASA's
Aviation Safety Program (AvSP) and is a UAS platform for the testing of experimental flight control laws and other vehicle dynamics research [1] [2]. Its current incarnation provides a dynamically scaled General Transport Model (GTM) aircraft controlled via a ground-based internal pilot uplink with a supplementary external pilot for take-off, landing, and safety maneuvering purposes. All flight data is transmitted via RF link to the Mobile Operations Station (MOS). The MOS contains the internal piloting and research personnel stations, houses the hardware on which the ground flight control system executes and uplinks the generated command output back to the aircraft [3]. Both data and control latency are characterized and factored into the design of the system.

This Phase-IV operational model was arrived at through a four-phase implementation schedule. This phased approach (Table 1) allowed functionality and support infrastructure to be built upon the previous phase and reduce the project implementation risk.

\begin{tabular}{|l|c|c|c|c|}
\hline Element & P-I & P-II & P-III & P-IV \\
\hline External Pilot & $\mathrm{X}$ & $\mathrm{X}$ & $\mathrm{X}$ & $\mathrm{X}$ \\
\hline Dyn. Scaled Vehicle & & & & $\mathrm{X}$ \\
\hline FCU Data System & & $\mathrm{X}$ & $\mathrm{X}$ & $\mathrm{X}$ \\
\hline MOS & & & $\mathrm{X}$ & $\mathrm{X}$ \\
\hline Internal Pilot & & & & $\mathrm{X}$ \\
\hline
\end{tabular}

Table 1. AirSTAR Phased Implementation

In Phase-IV operations (Figure 1), the External Pilot performs the take-off procedure, gains appropriate altitude, hands off control of the aircraft to the Internal Pilot in the MOS who performs the research maneuvers, and then the External Pilot reestablishes control and performs the landing maneuver. 


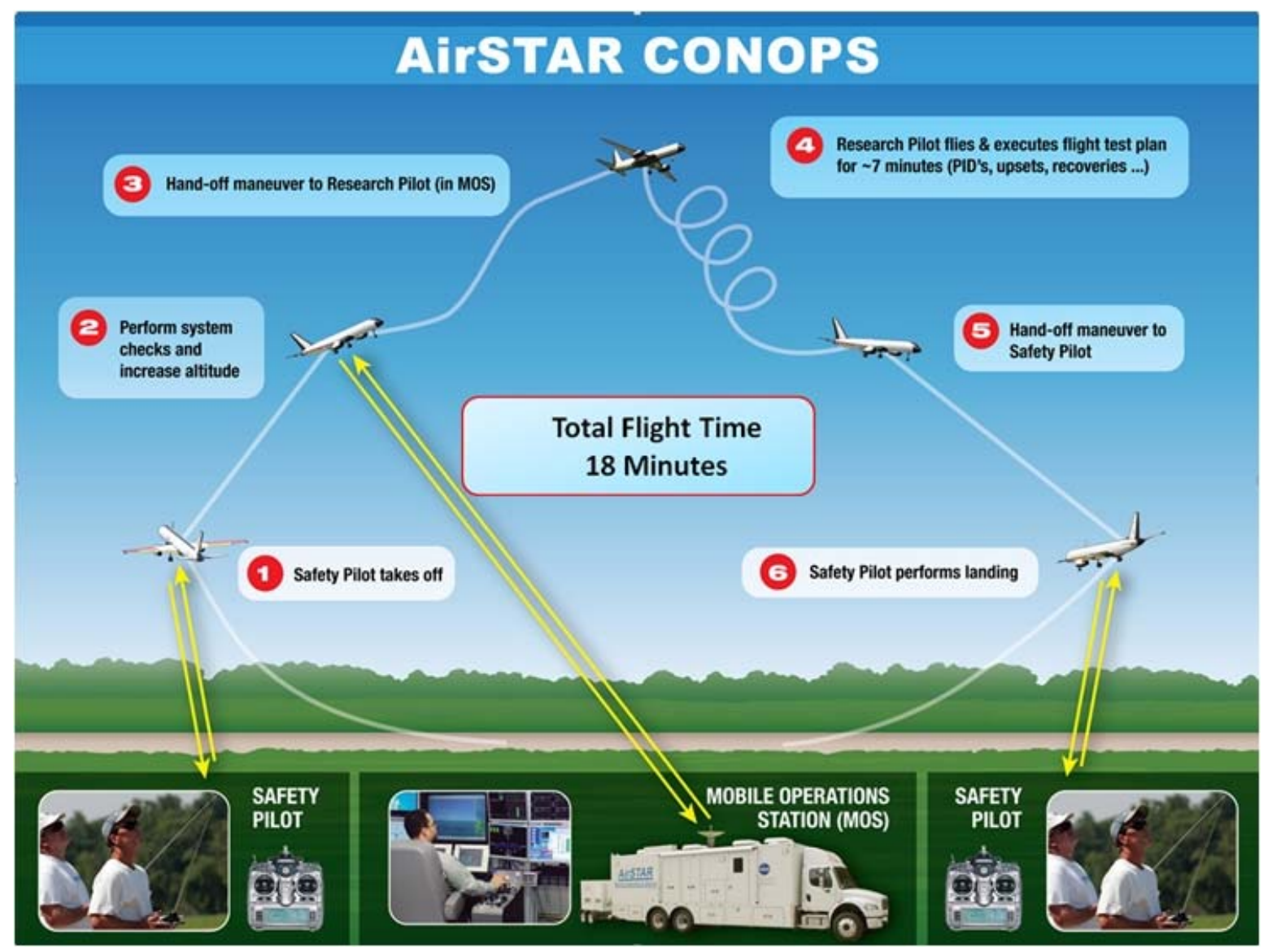

Figure 1. AirSTAR Phase-IV Concept of Operations

The initial Phase-I work encompassed developing the procedures and infrastructure necessary for deploying with the aircraft for operations while flying with only External Pilot control. Phase-II added the data system and telemetry downlink into the system and procedures, but still was only operating using an External Pilot. The Phase-III work integrated the MOS as the receiving station for telemetry data, but the internal piloting station was not used to control the aircraft, instead still relying only on the External Pilot. Finally, Phase-IV integrated the Internal Pilot into the operations as described above, and integrated the system into the actual dynamically scaled General Transport Model. The review of the hardware implementation and retrofit begins with a discussion of the Phase-III to Phase-IV transition.

\section{Phase-III to Phase-IV(a) Transition}

The AirSTAR Phase-III implementation was marked by the successful downlink of telemetry data to the MOS while flying the aircraft under control of an RC External Pilot. Prior to Phase-III the data had been transmitted to a laptop station on the ground. The MOS allowed greater real-time and post data analysis in the field, increasing the flight turn-around and mission planning speed. Planned flight maneuvers were able to be altered based on data reviewed on site immediately following a research flight. Missing from Phase-III is the Internal Pilot uplink command to aircraft. The lack of Internal Pilot control results in a simple servo control path with the servos connected directly to the External Pilot receiver unit (Figure 2). 

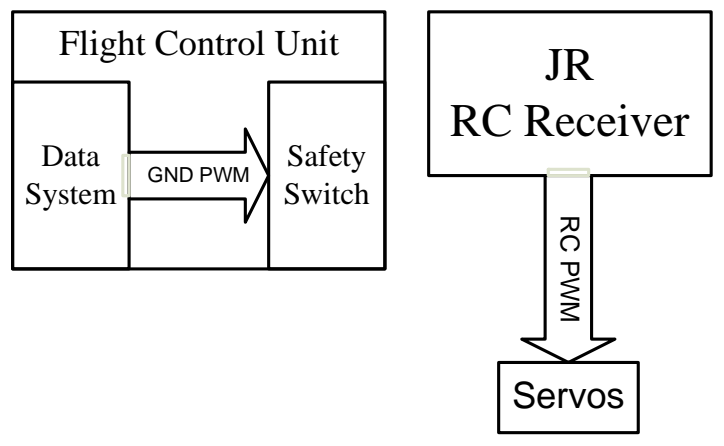

Figure 2. Phase-III Servo Control Path

Data telemetry, provided by the Flight Control Unit (FCU) (RF link omitted for brevity), is a completely separate power system and does not integrate with servo control at all in Phase-III operations. The FCU Data System (Figure 3) consists of three major components. These are the analog sampling hardware (omitted from command path illustrations for brevity), the "data system" which commutates and decommutates uplink and downlink data, including uplinked Internal Pilot commands, and the servo command Safety Switch. The Safety Switch was not in use during Phase-III and was the major focus of implementation during the move to Phase-IV operations.

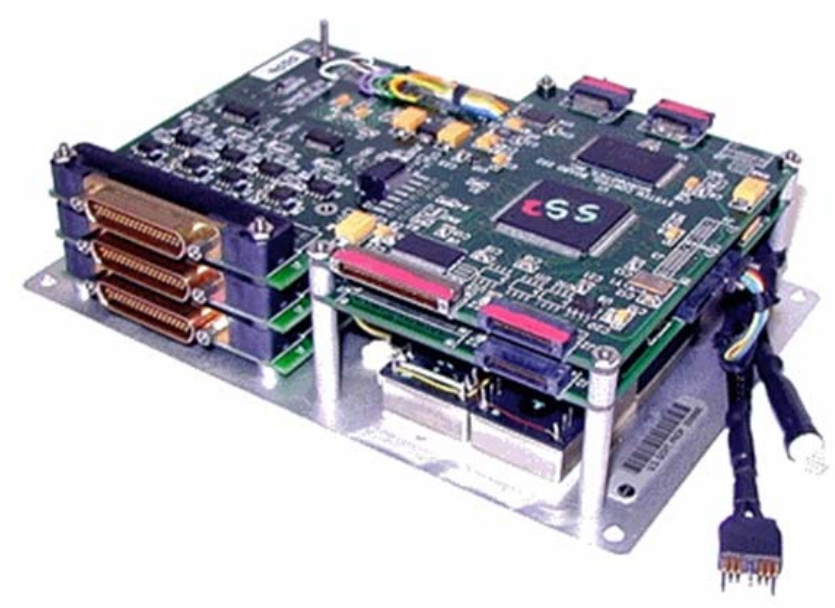

Figure 3. AirSTAR Flight Control Unit

The main challenge in acquiring successful research data in Phase-III was the sensitivity of the External Pilot RC receiver to externally generated electro-magnetic interference (EMI). FCU-generated EMI would cause the RC receiver to enter a failsafe condition due to a detected loss of link with the pilot's transmitter. This failsafe condition causes preset surface positions to be executed in order to bring the aircraft down with a minimum amount of ground distance coverage. Phase-III was using an older $72 \mathrm{MHz}$ RC system and in order to combat this problem a number of tactics were employed.

Anechoic chamber measurements of the aircraft's EMI characteristics were made to understand how different parts of the electronics contributed to the issue (Figure 4).

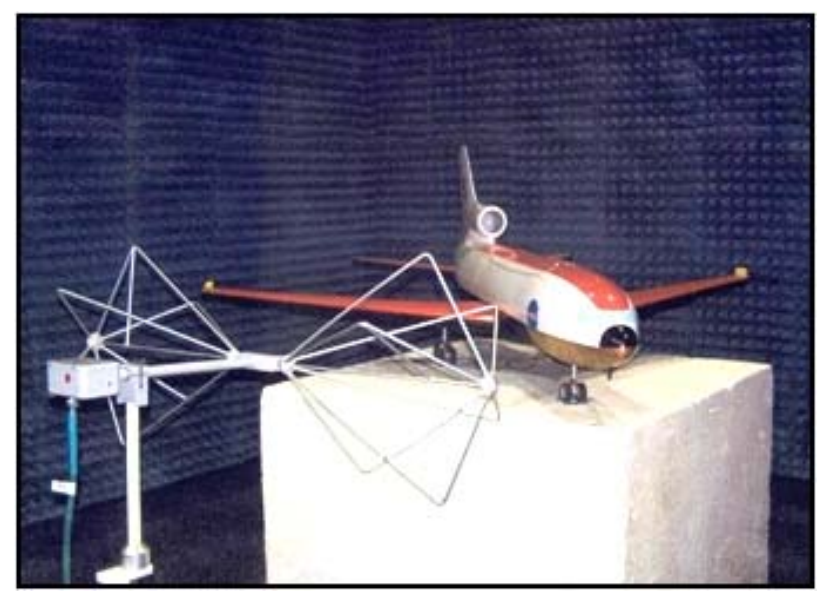

Figure 4. S-2 Model In Anechoic Chamber

Once the EMI characteristics of the airborne electronics were understood, channel selections for the $72 \mathrm{MHz} \mathrm{RC}$ receiver were made to avoid the most problematic frequency ranges. Improved shielding on the FCU was also employed to attempt to squelch emissions from the system (Figure 5).

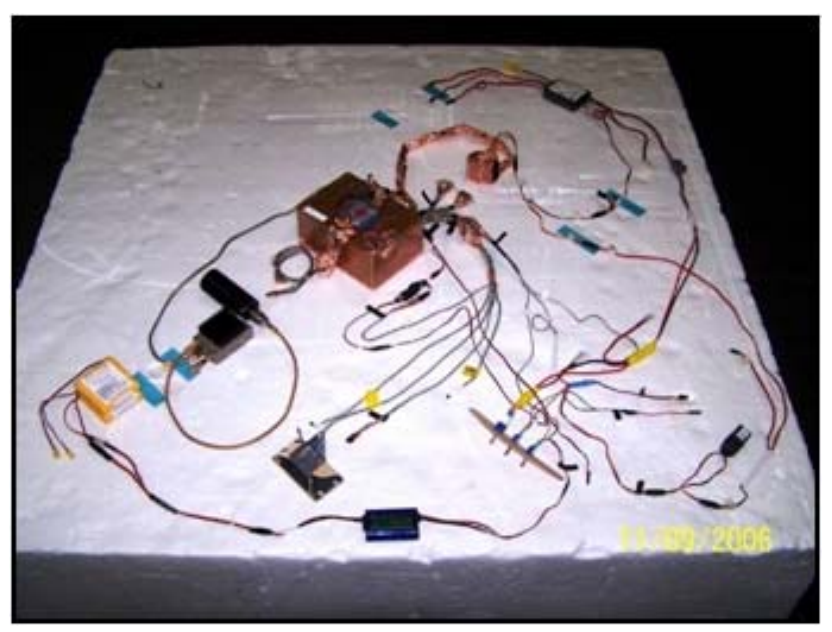

Figure 5. Shielded FCU In Anechoic Chamber 
Since the anechoic chamber results indicated that the FCU itself was the major contributor of EMI, a failsafe strategy to assure protection of the aircraft was employed. If the External Pilot RC receiver were to enter its failsafe condition, it would trigger a shutdown of the FCU in order to eliminate the EMI source and re-establish the External Pilot control link.

The hardware that detected the failsafe and triggered the shutdown was known as the FCU Kill Switch and was implemented using a parallel system of asynchronous RC-based switches (similar to those traditionally used for ignition switch applications). A pulse-width modulation (PWM) signal pulse time of $1 \mathrm{~ms}$ indicated a failsafe condition to trigger the system. The FCU Kill Switch was retrofitted into the existing power harness and worked well to protect the airframe during Phase-III deployments.

Moving from Phase-III to Phase-IV the milestone was to successfully fly the aircraft from the Internal Pilot station for actual research maneuvers. This affords more precise research control than is possible with the External Pilot so that more advanced research can be accomplished.

At a hardware level, Phase-IV necessitated the introduction of the FCU's previously unused hardware Safety Switch. The Safety Switch is able to switch between uplinked Internal Pilot commands and another servo command source. The switch is controlled by an external PWM signal from the External Pilot receiver. This makes the External Pilot the pilot-in-command since he controls which pilot is flying the aircraft. This changes the servo control path from the original Phase-III implementation to a new control path utilizing the Safety Switch (Figure $6)$.

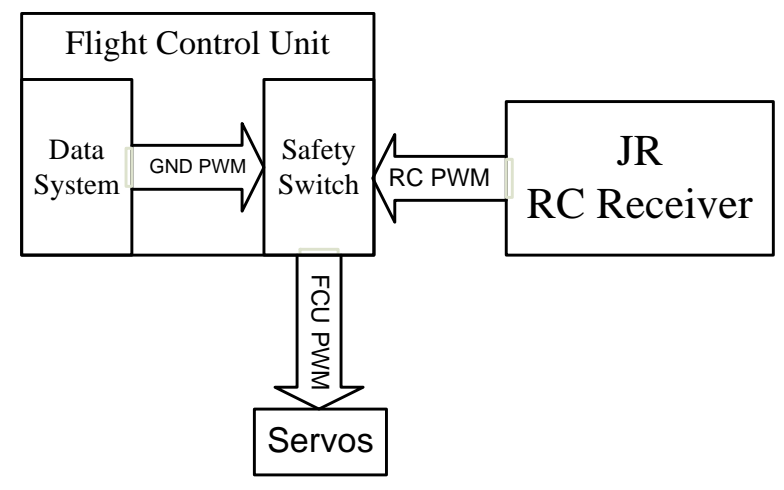

Figure 6. Phase-IV Concept Servo Control Path
Taking into account the Phase-III EMI failsafe strategy, the problem this new control path presents becomes apparent. All control systems now pass through the FCU Safety Switch, so the Internal and External piloting systems are no longer discrete systems. If the FCU is powered off in order to mitigate EMI then all pilots are severed from servo control, and specifically the External Pilot, the very piloting link the system needs to preserve.

Despite this, the decision was made to preserve the current EMI mitigation strategy moving forward through Phase-IV. This was done for both operational awareness purposes and uncertainty about the timeline for transitioning to a potentially more EMI-resistant External Piloting system. Obviously, this decision would require more changes and hardware retrofits into the existing avionics system.

The system would continue to use the FCU Safety Switch under nominal flight conditions. Nominal Internal-to-External Pilot handoffs, and vice versa, would occur in the FCU Safety Switch. Even in the case of FCU telemetry link failure (ie. uplink commands from the MOS are not reaching the data system), the External Pilot would be given command of the aircraft via the FCU Safety Switch and the FCU would remain operational. When the External Pilot RC link went into failsafe it would still power off the FCU, but this essentially requires the External Pilot commands to then be routed around, or bypass, the FCU. This creates the servo control path illustrated below (Figure 7).

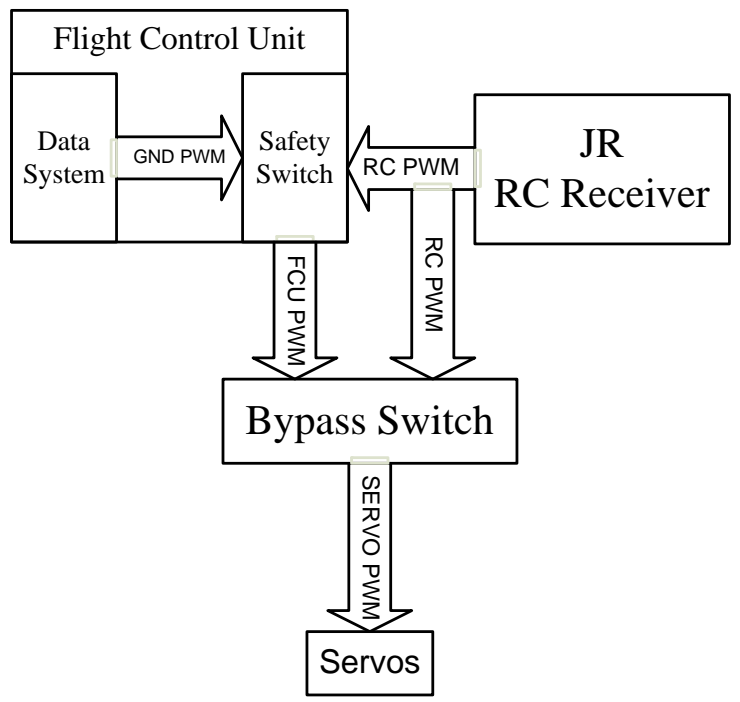

Figure 7. Servo Control Path w/ Bypass 
This second unit was known as the Bypass Switch, and in conjunction with the FCU Kill Switch enabled the transition of the existing failsafe strategy into Phase-IV operations.

As in Phase-III, the initial hardware implementation of this system was retrofitted into the existing avionics wiring harness. The Bypass Switch itself was implemented using a group of analog multiplexer ICs that were directly switched based on the failsafe state of the External Pilot receiver. At the same time, the Kill Switch was redesigned from the original RC switch-based design to a D-Flip Flop implementation so that the packaging could be minimized while retaining the same design (Figure 8). Even though the hardware implementation was refined, the two units still required two separate enclosures that added to the complexity of the avionics wiring harness itself and to the weight of the aircraft.

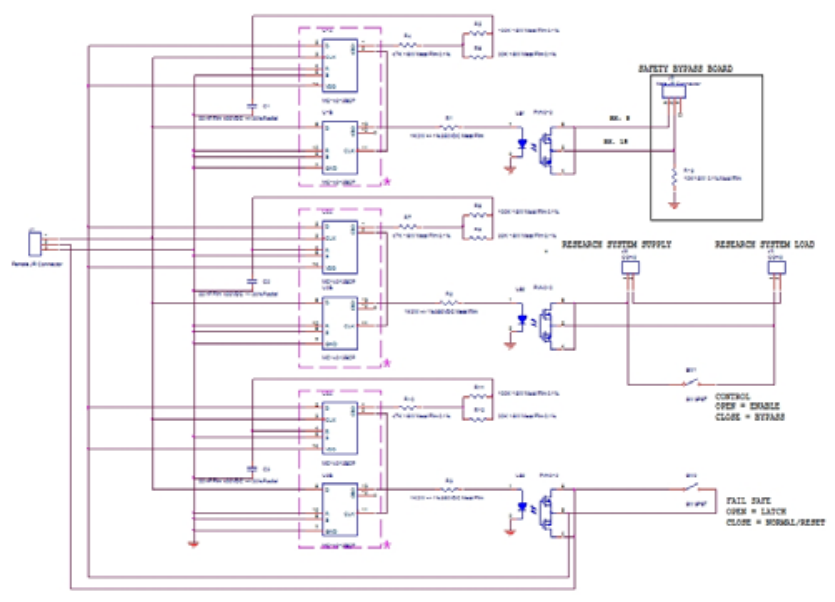

Figure 8. Kill Switch D-Flip Flop Implementation

This implementation of the FCU Kill Switch and the Bypass Switch flew on the initial Phase-IV flights that were done with the non-dynamically scaled S-2 vehicle (Figure 4).

\section{Phase-IV(a) to Phase-IV(b) Transition}

The initial transition into Phase-IV came to be known as Phase-IV(a) due to the fact that it was done using the non-dynamically scaled S-2 aircraft. After successful deployment of the full Phase-IV capability in this aircraft, the build-up of the same system in a dynamically scaled general transport model (GTM), T-2, became known as Phase-IV(b).

\section{Design Change Rationale}

A dynamically scaled aircraft can be mathematically related to its full-scale counterpart, allowing for flight research of larger vehicles in smaller models [4]. The nature of dynamically scaling an aircraft requires meeting metrics for both weight and CG of the of the scale model. The aircraft's propulsion and fuel systems set limits on airspeed, and the physical model itself sets limits on size and g-limits. Therefore, in the same way the weight of model must be carefully built so that at some point during flight the proper dynamic scaling of the aircraft under study is attained during which data points can be that made a repackaging of the data system necessary. Therefore, the prime motivation for the work on the GTM model's avionics harness was motivated by better controlling the weight and weight distribution of the electronics in the T-2 model (Figure 9). The final T-2 aircraft was a 5.5\% dynamically-scaled model weighing approximately 58 lbs., including fuel, at take-off, with a wingspan of 85 inches.

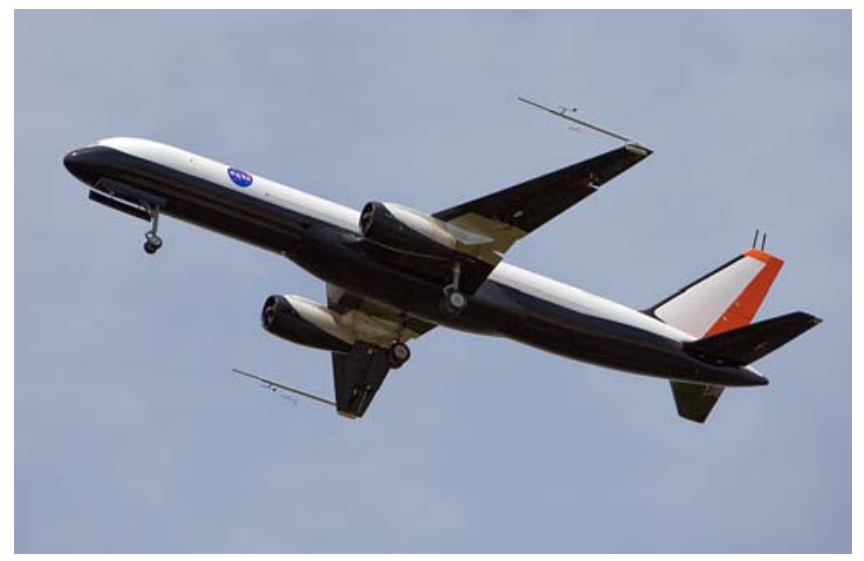

Figure 9. Dynamically Scaled GTM T-2 Model

Ease of access to system components for debugging was found to be an issue in the initial system as well, and this issue had a chance to be addressed during this fabrication effort. EMI mitigation was another primary driver behind the fabrication effort for the T-2 avionics, and there were some operational bugs in the initial retrofitted hardware that needed to be addressed. 
All of these considerations resulted in great attention being paid to three major areas of the new electronics build effort. First, power distribution and associated EMI considerations, second, the redesign of the existing Bypass Switch system, and finally, the actual packaging of the electronics, which follows closely from the first two design efforts.

\section{Power Distribution and EMI}

The power system in Phase-IV is completely powered by traditional RC model Lithium-Ion battery packs, and requires three discrete battery systems to operate. One powers the FCU Data System and other avionics, the second powers the Engine Control Units (ECU) for the turbine engines, and the third powers the External Pilot RC piloting system and the servos themselves.

Initially, no consideration was paid in the harness itself to isolating these systems. In fact, power regulation and distribution for everything, including the servos, was housed in the same enclosure as the data system Measurements of both actual noise generated by the servos and the general transient behavior of the current requirements of the servo system made it prudent to isolate the FCU Data System power from the RC piloting system and servos. To this end a grounding tree was devised to map out the power systems of the aircraft avionics.

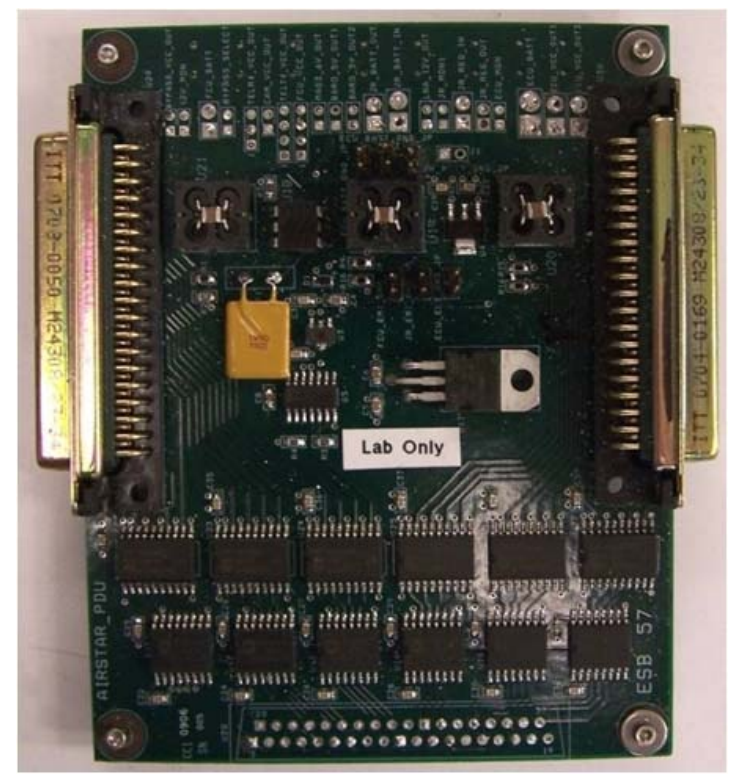

Figure 10. Power Distribution Unit PCB
Central to this grounding tree was a Power Distribution Unit (PDU) that was designed to house most of the power regulation in the aircraft (Figure 10). The PDU kept the power and ground rails of the data/sensor system isolated from the servos and ECU power systems, and served as the central distribution point for most aircraft power. Since it was so central to the system it was also designed to serve as the central connection point for all of the servos in the aircraft.

Major design components of the PDU included EMI filters on all battery input rails, and discrete separation of power regulation areas in the board design phase. Line drivers were also used on all servo command outputs in the case that servo cabling was to be long, since final placement of the PDU in the aircraft was not known until later in the design phase.

The FCU Data System was given its own enclosure, with only a small amount of voltage regulation necessary for sensor excitation and small logic needs actually being performed inside the enclosure. Fabrication and external connector positions were carefully chosen as to prevent power wiring internal to the enclosure from running near analog sampling hardware of the data system.

\section{Bypass \& Kill Switch Redesign}

During Phase-IV(a) post-deployment activities it was discovered that the retrofitted Kill and Bypass Switch system was extremely sensitive to system noise and power current transients. Sudden current draws, the result of large, fast, simultaneous servo movements across a number of aircraft servos would result in a drop of the servo power rail and the PWM command signal. While the servos themselves seemed resilient to these power transients, the RC switches used in the Kill Switch were not and would detect these lowered voltage-level PWM signals as actual zero percent duty-cycle pulses. This would result in the Kill Switch detecting a failsafe condition when, in fact, there was none.

Since the entire avionics system was to be repackaged, the decision was made to redesign the retrofitted system and integrate the Bypass Switch and Kill Switch into a single board system. Furthermore, the system would be fabricated so as to 
fit natively on the FCU Data System footprint and reside within the same enclosure.

The redesigned system was implemented using a VHDL design in a Xilinx Coolrunner XPLA3 CPLD device, and verified through test flights on a simple Ultrastick prop aircraft.
The major VHDL modules (Figure 11) developed for the system implementation were a pulse-length detector (PWM_COUNTER.vhd), a failsafe pulse counter (FAILSAFE_COUNTER.vhd), a generic switching module (SWITCH.vhd), and the switch's controlling state-machine (BYPASS_STATEMACHINE.vhd).

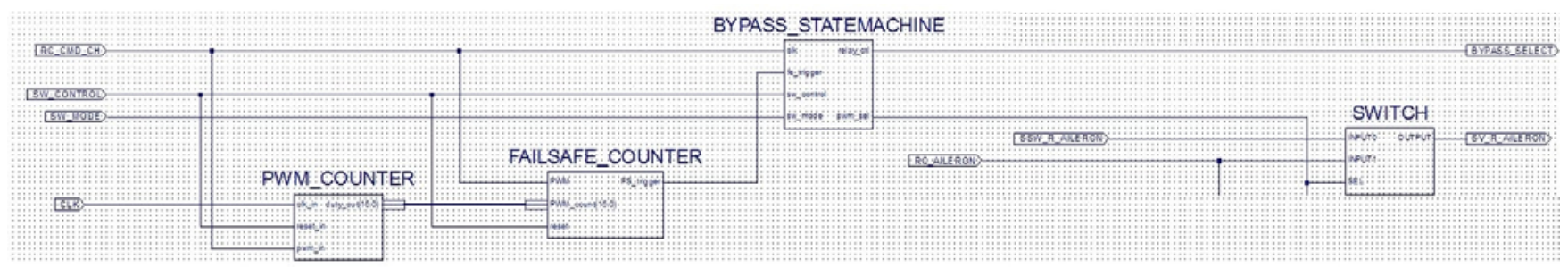

Figure 11. Phase-IV(b) Bypass Switch VHDL Configuration

All of the modules are synchronous with the exception of the switching module itself. The pulselength detector generates a 16-bit count to indicate the pulse-length of the current pulse-width modulation signal. This count is fed into the failsafe pulse counter that compares it to a static metric value equivalent to $1 \mathrm{~ms}$ in order to determine if the pulse length indicates a failsafe condition. The pulse counter keeps a count of the train of failsafeindicating pulses until either a count threshold is reached, or the pulses stop indicating failsafe.

If the count threshold is reached first, the trigger from the failsafe pulse counter tells the state machine that the system has entered failsafe mode. If the pulse lengths stop indicating failsafe before the threshold is reached, the failsafe pulse counter resets its count and restarts waiting for pulses that indicate failsafe. The system state machine gets no indication of a failsafe event.

This count threshold was derived to require failsafe to be indicated for approximately 0.25 seconds before initiating the failsafe trigger. This allowed the system to be robust against the falsepositive failsafe indications that would trigger the original system.

The state machine module also controls the operational state of the Bypass Switch via the externally operable switches on the FCU enclosure. One switch simply turns the Bypass Switch on or off. The avionics system essentially operates as if the Bypass Switch was not present when the Bypass
Switch is turned off. This was a useful mode during lab testing when External Pilot link failures were not a concern.

The second switch determines the latching behavior of the Bypass Switch. When in latching mode the Bypass Switch permanently latches the system into External Pilot bypass mode - turning off the FCU Data System, and routing External Pilot commands directly to the servos - regardless of whether the External Pilot command link recovers later.

When in non-latching mode the Bypass Switch will move out of its bypass state - turning the FCU Data System back on, and using the FCU Switch servo outputs - when the External pilot link returns to a standard operational state. Non-latching mode is used only during lab tests, integration testing of the system and on the flight line during pre-flight and thru-flight procedures. The pre-flight and thru-flight checklists ensure the aircraft is returned to latching mode prior to flight.

Finally, the actual layout of the redesigned Bypass Switch system was designed to work with our developed Power Isolation and EMI strategy (Figure 12). The Bypass Switch has a necessary interface between the External Piloting power system and the FCU Data System power system, and proper isolation was necessary.

EMI filters, as found in the PDU, are used on this board as well for the power inputs. External Pilot servo commands enter from the bottom of the 
board and pass through the array of isolation devices before going to the system logic that runs from the FCU Data System power rail.

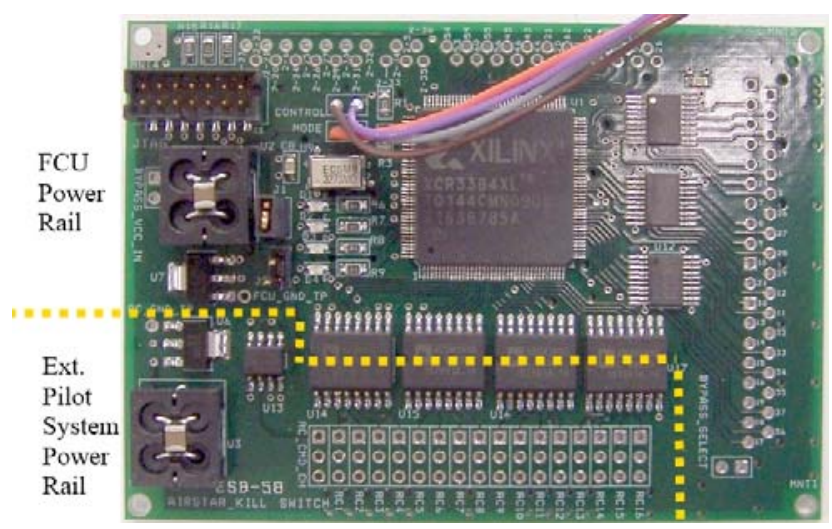

Figure 12. P-IV Bypass Switch Board

The FCU Data System Safety Switch outputs enter from the top of the board with no isolation necessary since the components share the same power system. The selected output commands exit out the right of the board, passing through line drivers due to the cable length between the Safety Switch and the PDU, where the servo commands are then all distributed to the servos. In the PDU, isolation is also provided where the servo command signals pass from the FCU power system into the External Pilot and Servo power system.

\section{Avionics Packaging Redesign}

All of the effort expended in the redesign would be meaningless if the packaging of the electronics was not addressed as well. The main issues addressed were cable shielding, individual component construction, and component layout in the aircraft.

Single-ended grounded shielding was utilized throughout the system as a guard against EMI emissions. The primary enclosures for the two main components, the FCU and the PDU, were redesigned to accommodate better connectors and have proper mounting in the aircraft (Figure 13) (Figure 14).

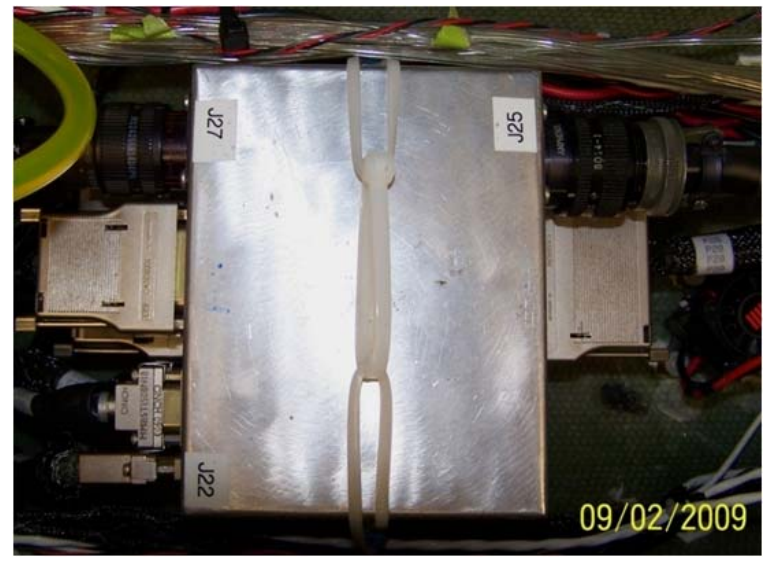

Figure 13. PDU Enclosure, T-2 Mount

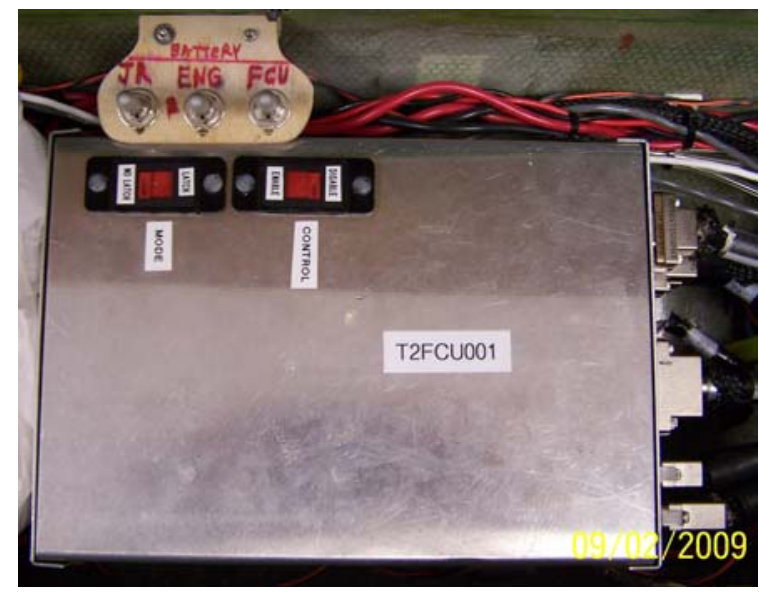

Figure 14. FCU Enclosure, T-2 Mount

Many of the initial Phase-III enclosures had umbilical style cabling that could not be removed, making it difficult to replace discrete components in the aircraft. This was mainly due to the way in which the build-up was done, but the move to T-2 allowed for this repackaging, as illustrated, which allowed both easier troubleshooting of the system, and simpler replacement of components.

Finally, a good deal of the EMI-instigated problems with the External Pilot link were mitigated by moving away from the older $72 \mathrm{MHz}$ External Pilot hardware and to the newer $2.4 \mathrm{GHz}$ Spread Spectrum hardware available from the RC aircraft vendors. The EMI noise from the FCU did not affect this higher frequency range nearly as much as it did the $72 \mathrm{MHz}$ system. 


\section{Phase-IV(b) System Deployment}

The redesigned T-2 avionics hardware system was deployed for the first time at Wallops Island, VA in September, 2009. The system to date has flown a total of fifty-eight research flight missions.

\section{Conclusion and Future Work}

The effort to retrofit hardware into the Phase-IV FCU Data System allowed the stand-up of a fully functional Phase-IV capability for research utilizing a $5.5 \%$ dynamically scaled GTM aircraft. This avionics work was necessary to meet safety assurance measures required by the AirSTAR project for flight, and successfully integrated both the Internal Pilot and the External Pilot, as pilot-in-command, together to form the backbone of the research operations provided by the AirSTAR UAS system.

Future work sees AirSTAR capability expanding even further as the project is designing and implementing a new system to enable beyond visual range operations utilizing exclusive Internal Pilot control in the MOS. This will allow the expansion of the operational and hazard areas of the system and allow for a wider range of research applications for the system.

\section{Acknowledgments}

The author would like to thank the entire research and engineering team involved in the development of the AirSTAR test bed for their continued work in growing the capability of this novel UAS platform.

\section{References}

[1] Bailey, R.M., Hostetler, R.W., Barnes, K.N., Belcastro, Christine M., and Belcastro, Celeste M., "Experimental Validation: Subscale Aircraft Ground Facilities and Integrated Test Capability," AIAA Guidance, Navigation, and Control Conference, AIAA, San Francisco, CA, 2005.

[2] Foster, John V. et al; "Dynamics Modeling and Simulation of Large Transport Airplanes in Upset Conditions," AIAA-2005-5933, AIAA Guidance, Navigation and Control Conference, AIAA, San Francisco, CA, 2005.

[3] Murch, A.M., “A Flight Control System Architecture for the NASA AirSTAR Flight Test Infrastructure,” AIAA-2008-6990, AIAA Guidance, Navigation, and Control Conference and Exhibit, Honolulu, Hawaii, Aug. 18-21, 2008.

[4] Wolowicz, C.H., Bowman, Jr., J.S., and Gilbert, W.P., "Similitude Requirements and Scaling Relationships as Applied to Model Testing”, NASA TP-1435, 1979.

\section{1st Digital Avionics Systems Conference}

October 16-20, 201 